\title{
Universiteit
}

Leiden

The Netherlands

\section{Speciation through the learning of habitat features}

Beltman, J.B.; Haccou, P.

\section{Citation}

Beltman, J. B., \& Haccou, P. (2005). Speciation through the learning of habitat features.

Theoretical Population Biology, 67(3), 189-202. doi:10.1016/j.tpb.2005.01.001

Version: $\quad$ Publisher's Version

License: $\quad$ Licensed under Article 25fa Copyright Act/Law (Amendment Taverne)

Downloaded from: https://hdl.handle.net/1887/3238718

Note: To cite this publication please use the final published version (if applicable). 


\title{
Speciation through the learning of habitat features
}

\author{
J.B. Beltman ${ }^{\mathrm{a}, \mathrm{b}}$, P. Haccou ${ }^{\mathrm{a}, *}$ \\ ${ }^{a}$ Section Theoretical Biology, Institute of Biology, Leiden University, Kaiserstraat 63, 2311 GP Leiden, The Netherlands \\ ${ }^{\mathrm{b}}$ Section Behavioural Biology, Institute of Biology, Leiden University, Kaiserstraat 63, 2311 GP Leiden, The Netherlands
}

Received 6 November 2004

\begin{abstract}
Learning of environmental features can influence both mating behaviour and the location where young are produced. This may lead to speciation in three steps: (i) colonization of a new habitat, (ii) genetic divergence of the two groups by adaptation to the habitats, and (iii) a decrease of genetic mixing between the lineages (similar to reinforcement). In a previous paper we showed that steps (i) and (ii) occur readily for a wide range of fixed mating and habitat preferences. Here, we study whether this can ultimately lead to speciation through selective changes in these preferences.

We show that this indeed occurs, and, furthermore, it is very general: for a large class of models there is selection toward producing young more frequently in the natal habitat. Once habitat preference is strong, there is selection toward stronger assortative mating. Even when steps (i) and (ii) initially fail, genetic divergence may succeed at a later evolutionary stage, after which a decrease of genetic mixing completes speciation. Our results show that speciation by the learning of habitat features is an extremely effective mechanism.
\end{abstract}

(C) 2005 Elsevier Inc. All rights reserved.

Keywords: Learning; Speciation; Habitat preference; Reinforcement

\section{Introduction}

The potential impact of learning on evolutionary processes has been noted only occasionally (e.g., ten Cate and Bateson, 1988; Grant and Grant, 1997; Irwin and Price, 1999; Owens et al., 1999; ten Cate and Vos, 1999; Slabbekoorn and Smith, 2002), and the amount of theoretical studies investigating it is limited (e.g., Laland, 1994a,b; Aoki et al., 2001; Ellers and Slabbekoorn, 2003; Beltman et al., 2003, 2004). Studies of speciation usually focus on the geographical mode of speciation (see Mayr, 1942, 1963; Schilthuizen, 2001; Turelli et al., 2001; Gavrilets, 2004; Coyne and Orr, 2004). Indeed, a multitude of models has been produced

\footnotetext{
${ }^{*}$ Corresponding author. Section Theoretical Biology, Institute of Biology, Leiden University, Kaiserstraat 63, 2311 GP Leiden, The Netherlands. Fax: + 31715274900

E-mail addresses: beltman@rulsfb.leidenuniv.nl (J.B. Beltman), Haccou@rulsfb.leidenuniv.nl (P. Haccou).
}

to investigate speciation in the presence of geneflow (e.g., Maynard Smith, 1966; Pimm, 1979; Kawecki, 1997; Dieckmann and Doebeli, 1999; Higashi et al., 1999; Kondrashov and Kondrashov, 1999; Gavrilets, 2000; van Doorn and Weissing, 2001; Gavrilets and Waxman, 2002; Fry, 2003), ignoring the potential role of learning.

Learning can influence speciation when the mating behaviour of individuals depends on their experience, which happens in many species. Sexual imprinting (reviewed in ten Cate and Vos, 1999), the acquisition of mate preferences through learning the characteristics of parents or siblings early in life (Lorenz, 1937), provides an example. Mating behaviour can also be influenced by the learning of environmental (habitat) features. For instance, specialist brood-parasitic Viduidae learn the songs of their foster species. Males learn to produce these songs (Payne et al., 1998), and females use the songs they heard early in life (i) to find suitable mates, and (ii) to locate host nests (Payne et al., 2000). 
In this example the habitats are the foster species, and the learned habitat features their songs. The learning of habitat features also occurs in other animal species such as fish, insects and mammals. West-Eberhard (2003) and Beltman et al. (2004) discuss several examples, but a more extensive review is provided by Davis and Stamps (2004), who refer to this phenomenon as "natal habitat preference induction". They suggest that it has been understudied, in part because scientists working with different taxa have used different terms to describe it (e.g., Hopkins' host selection principle, or habitat imprinting). Well-known examples are fish species that imprint olfactorily at a young age on their local habitat, and use this learned information as adults for homing (e.g., salmon), or insects that prefer to feed on the food plant they have experienced since their birth. The learned host, habitat, or food features can influence both mating behaviour (here referred to as mating preference) and the location where young are produced (here referred to as habitat preference).

As a consequence of habitat imprinting, females normally produce their young in the same habitat as they grew up in. They may, however, accidentally produce their young in another habitat. The frequency of such an event will depend on the strength of the females' preference for their natal habitat. The young resulting from "accidents" will learn features of the new habitat. Because of this, they will most likely mate with other individuals exploiting the habitat, and adult females will tend to produce their young in the new habitat.

In a previous paper (Beltman et al., 2004) we argued that, through such processes, the colonization of a new habitat may eventually lead to speciation. We used a gene-culture coevolutionary model to study this. It was assumed that population density is regulated separately in each of the two habitats, and that the viability of an individual depends on its genotype as well as on the habitat it exploits. Starting from a population that is specialized on the exploitation of a particular habitat, we studied under what circumstances colonization of a new habitat is possible and whether genetic differences between the subpopulations can arise. We showed that under a wide range of conditions, genetic divergence indeed occurs.

However, in that analysis, the strengths of both the habitat and the mating preference were assumed to be fixed traits. In reality, these traits will be under selective pressure. Furthermore, as long as these preferences are not absolute, there is no complete reproductive isolation. Therefore, our previous results imply only that the first steps toward speciation can occur when individuals imprint on habitat features, but it cannot be concluded that eventually this will indeed lead to divergence into different species. To examine that, we need to study evolutionary changes in habitat and mating preferences.
Selection for changes in habitat preferences is equivalent to selection for migration modification as studied by Karlin and McGregor (1972), Balkau and Feldman (1973), and Karlin and McGregor (1974). However, these authors only consider situations where mating occurs within the habitats, which implies that mating preferences are assumed to be absolute. As a consequence, reproductive isolation is accomplished as soon as the migration fraction is zero. We allow mating frequencies to depend on a mating preference as well as the availability of potential partners from different habitats, and consider evolutionary changes in the strength of this preference.

In the present paper we examine the direction of selection on the two traits by means of the selection differentials. Our main assumptions are that viability selection takes place after density regulation, that density regulation is such that the attractors are fixed points, and that viability selection acts in different directions in the subpopulations. First, we show that under very general conditions there is selection toward an increased habitat preference, regardless of the amount in which different habitats are exploited initially. Subsequently, we show that in a large class of models there is selection toward increased assortative mating as soon as habitat preferences are sufficiently high. These findings together imply that speciation will occur whenever a genetic polymorphism for viabilities in different habitats evolves at high habitat preferences. We examine the conditions under which this occurs for the model discussed by Beltman et al. (2004). We find that, even in cases where successful colonization and genetic divergence does not occur initially, speciation often takes place in the long run. Thus, speciation assisted by the learning of habitat features is an extremely effective mechanism.

\section{Description of the model}

As before, we consider two habitats, $A$ and $B$. The viability within a habitat is determined by one diploid locus with two alleles. The three possible genotypes are denoted by $g_{A} g_{A}, g_{A} g_{B}$, and $g_{B} g_{B}$. Individuals are further characterized by a cultural trait that is determined by the habitat that they are born into, $c_{A}$ or $c_{B}$. We assume that there is female demographic dominance, and dynamics of females and males are equal, so that it suffices to follow only female dynamics. Generations are discrete and non-overlapping. It is assumed that mating is polygynous, and female preferences depend on male frequencies as well as cultural traits. After or during production of young there is local density regulation, and then viability selection takes place. We here consider a wider class of gene-culture coevolution models than in Beltman et al. (2004) in that we 
allow more general forms of density dependence and mate preference.

We denote the probability that a female that was born in habitat $x$ mates with a male from the same habitat by $\rho_{x}(\alpha)$, where $\alpha$ is the strength of the mating preference. These functions are assumed to be non-negative and non-decreasing in $\alpha$, approaching one as $\alpha$ tends to infinity.

Females produce their young in the same habitat as they were born in with probability $1-p$ (we refer to $1-p$ as the habitat preference). In each habitat there is local density regulation, which is determined by a function $Q_{x}$ that depends on the density in habitat $x$. We restrict our attention to functions $Q_{x}$ of such a form that the only population dynamical attractors are fixed points.

The densities of reproductive adult individuals of the six possible combinations of cultural trait and genotype are denoted by $N_{i}(i=1 \ldots 6$, see summary in Table 1$)$. Furthermore, we denote the total densities in each habitat by

$N_{A}=N_{1}+N_{2}+N_{3}$,

$N_{B}=N_{4}+N_{5}+N_{6}$.

Individuals are subject to viability selection, depending on their genotype and the habitat they exploit. In habitat $A$, individuals carrying more $g_{A}$ alleles have a higher viability than other individuals, while in habitat $B$ this is the case for individuals carrying more $g_{B}$ alleles. We denote the viability of the types by $w_{i}\left(w_{1}\right.$ is the viability of $c_{A} g_{A} g_{A}$ individuals, $w_{2}$ that of $c_{A} g_{A} g_{B}$ individuals, etc.). The parameters $w_{i}$ can range from 0 (no individuals of this type survive) to 1 (all individuals of this type survive).

The model described above is a generalization of that used by Beltman et al. (2004). There we used the following specific forms of the functions $\rho_{x}(\alpha)$ and $Q_{x}$ :

$\rho_{A}(\alpha)=\frac{\alpha N_{A}}{\alpha N_{A}+N_{B}}, \quad \rho_{B}(\alpha)=\frac{\alpha N_{B}}{\alpha N_{B}+N_{A}}$,

and

$Q_{A}=\frac{E}{1+K E\left((1-p) N_{A}+p N_{B}\right)}$,

Table 1

The different types of individuals

\begin{tabular}{llll}
\hline Type & Density & Cultural trait & Genetic trait \\
\hline 1 & $N_{1}$ & $c_{A}$ & $g_{A} g_{A}$ \\
2 & $N_{2}$ & $c_{A}$ & $g_{A} g_{B}$ \\
3 & $N_{3}$ & $c_{A}$ & $g_{B} g_{B}$ \\
& & & \\
4 & $N_{4}$ & $c_{B}$ & $g_{A} g_{A}$ \\
5 & $N_{5}$ & $c_{B}$ & $g_{A} g_{B}$ \\
6 & $N_{6}$ & $c_{B}$ & $g_{B} g_{B}$ \\
\hline
\end{tabular}

$Q_{B}=\frac{E}{1+K E\left(p N_{A}+(1-p) N_{B}\right)}$,

where $E$ corresponds to female fertility, and $K$ is a positive parameter that determines the carrying capacity (note that a lower $K$ implies a higher carrying capacity). Our present results are, however, valid for any functions that meet the conditions specified previously.

We denote the frequency of allele $g_{A}$ among those with cultural trait $c_{A}$ and $c_{B}$ by respectively $g$ and $f$, i.e.

$g=\frac{N_{1}+\frac{1}{2} N_{2}}{N_{A}}, \quad f=\frac{N_{4}+\frac{1}{2} N_{5}}{N_{B}}$.

Furthermore, let $\beta_{x}$ be the probability that a female who was born in habitat $x$ produces offspring with paternal allele $g_{A}$, i.e.

$\beta_{A}=\rho_{A}(\alpha) g+\left(1-\rho_{A}(\alpha)\right) f$,

$\beta_{B}=\rho_{B}(\alpha) f+\left(1-\rho_{B}(\alpha)\right) g$.

In Appendix $A$ it is shown that the dynamics of this system are fully described by a set of recurrence equations for the total densities in each habitat $\left\{N_{A}\right.$, $\left.N_{B}\right\}$ :

$\left(\begin{array}{c}N_{A} \\ N_{B}\end{array}\right)^{\prime}=\left(\begin{array}{cc}(1-p) Q_{A} w_{A A} & p Q_{A} w_{A B} \\ p Q_{B} w_{B A} & (1-p) Q_{B} w_{B B}\end{array}\right)\left(\begin{array}{c}N_{A} \\ N_{B}\end{array}\right)$,

and a pair of recurrence equations for the frequencies of allele $g_{A}$ in each habitat $\{g, f\}$ :

$g^{\prime}=\frac{Q_{A}}{N_{A}^{\prime}}\left\{(1-p) w_{A A, 1} N_{A}+p w_{A B, 1} N_{B}\right\}$,

$f^{\prime}=\frac{Q_{B}}{N_{B}^{\prime}}\left\{p w_{B A, 1} N_{A}+(1-p) w_{B B, 1} N_{B}\right\}$,

where

$$
\begin{aligned}
w_{A A}= & \left(w_{1} \beta_{A}+w_{2}\left(1-\beta_{A}\right)\right) g \\
& +\left(w_{2} \beta_{A}+w_{3}\left(1-\beta_{A}\right)\right)(1-g), \\
w_{A A, 1}= & \left(w_{1} \beta_{A}+\frac{1}{2} w_{2}\left(1-\beta_{A}\right)\right) g+\left(\frac{1}{2} w_{2} \beta_{A}\right)(1-g) .
\end{aligned}
$$

The expressions for $w_{A B}$ and $w_{A B, 1}$ are found by replacing $\beta_{A}$ by $\beta_{B}$ and $g$ by $f$. The expressions for $w_{B A}$ and $w_{B A, 1}$ are found by replacing $w_{1}, w_{2}$ and $w_{3}$ by respectively $w_{4}, w_{5}$ and $w_{6}$. Finally, the expressions for $w_{B B}$ and $w_{B B, 1}$ are found by replacing the $w_{i}(i=1,2,3)$ as well as $\beta_{A}$ and $g$.

\section{Results}

We study the direction of selection on $\alpha$ and $p$ for the situation that

$w_{1}>w_{2}>w_{3}$ and $\quad w_{6}>w_{5}>w_{4}$. 
To do this we use the so-called adaptive dynamics approach (Metz et al., 1996; Dieckmann and Law, 1996; Geritz et al., 1998). This implies that we assume that the population is large, and that there are no other factors affecting mate choice besides the mating preference. Furthermore, it is assumed that mutation steps are small, so that mutants closely resemble the resident type, and that mutations are very rare, so that ecological and evolutionary time scales are separated. The last assumption entails that in-between mutation events the population dynamics reaches its attractor, which is in our case an equilibrium. The course of evolution can then be studied by considering which rare mutant types can establish themselves in a population of residents, which of these invasions will lead to replacement of the resident population, and what will be the outcome of a series of such substitution events.

We thus consider a population with resident trait values of the parameters (denoted by $p_{r}$ and $\alpha_{r}$ ) at its population dynamical equilibrium. Then we calculate the initial growth rate of a rare mutant type with a slightly different value of these traits. If this rate is larger than the growth rate of the resident population (which equals one since it is in equilibrium) the mutant type has a non-zero probability to establish itself. In the terminology of quantitative genetics we study the selection differentials of $\alpha$ and $p$.

By applying the results of Geritz et al. (2002) to our model, it can be shown that mutants that are able to invade will replace the resident population, as long as the process is not in the vicinity of so-called evolutionary branching points. Such branching points may occur if there is mutual invadability. This means that a mutant, say of type 1, can invade a population consisting of a resident type, here called 2 and that the reverse is also possible: mutants of type 2 can invade a resident population of type 1 . It turns out, however, that such situations do not occur here. Thus the selection differentials will tell us the course of evolution.

We only consider situations where the total population does not go extinct, i.e. the total density $N_{A}+N_{B}$ remains positive. There are several possibilities: when at equilibrium only allele $g_{A}$ or $g_{B}$ is present in the population, we will speak of a (genetically) monomorphic population. When both alleles are present we will call the population polymorphic.

We study the selection differentials of $p$ and $\alpha$ separately. While the evolutionary trajectory may be affected by correlations between these traits, this does not affect our main conclusions about the possibility of speciation.

\subsection{Selection on $p$}

We consider the effect of small mutation steps in the value of $p$. When mutants are rare, density-dependent components are determined by the resident population. Therefore, the initial growth rate of the mutant population equals the dominant eigenvalue of the matrix in Eq. (6), with $p$ equal to the mutant value $p_{m}$, whereas $Q_{x}$ and the $w_{x y}$ depend on the resident value $p_{r}$. We only consider situations where $p_{r} \neq 0$ or 1 . This eigenvalue equals

$\lambda=\frac{b+\sqrt{D}}{2}$,

with

$$
\begin{aligned}
D & =b^{2}-4 c, \\
b & =\left(1-p_{m}\right)\left(Q_{A} w_{A A}+Q_{B} w_{B B}\right), \\
c & =Q_{A} Q_{B}\left(\left(1-p_{m}\right)^{2} w_{A A} w_{B B}-p_{m}^{2} w_{B A} w_{A B}\right) .
\end{aligned}
$$

We will show that the derivative of $\lambda$ to $p_{m}$, evaluated at the value $p_{m}=p_{r}$ is negative, which means that there is selection in favour of a stronger habitat preference (toward smaller $p$ ). This can easily be shown to be equivalent to

$$
\left(\sqrt{D} \frac{\partial b}{\partial p_{m}}+b \frac{\partial b}{\partial p_{m}}-2 \frac{\partial c}{\partial p_{m}}\right)_{p_{m}=p_{r}}<0 .
$$

Since the resident population is at its equilibrium, $b+$ $\sqrt{D}$ must equal 2 when $p_{m}$ equals $p_{r}$, so the inequality becomes

$$
\left(\frac{\partial b}{\partial p_{m}}-\frac{\partial c}{\partial p_{m}}\right)_{p_{m}=p_{r}}<0
$$

The derivative of $b$ equals $-b /\left(1-p_{m}\right)$. From $b+\sqrt{D}=$ 2 it further follows that $b$ equals $1+c$, so the left-hand side of the inequality equals

$$
\left(\frac{-1}{1-p_{m}}(1+c)-\frac{\partial c}{\partial p_{m}}\right)_{p_{m}=p_{r}} .
$$

Substituting the expressions for $c$ and its derivative gives, after some rearranging,

$Q_{A} Q_{B}\left\{\left(1-p_{r}\right)^{2} w_{A A} w_{B B}+p_{r}\left(2-p_{r}\right) w_{B A} w_{A B}\right\}<1$.

From Eq. (6) we can derive that at the equilibrium of the resident population

$Q_{A}=\frac{N_{A}}{\left(1-p_{r}\right) w_{A A} N_{A}+p_{r} w_{A B} N_{B}}$,

$Q_{B}=\frac{N_{B}}{\left(1-p_{r}\right) w_{B B} N_{B}+p_{r} w_{B A} N_{A}}$.

Substituting this and rearranging gives

$\frac{w_{A A}}{w_{A B}} N_{A}^{2}+\frac{w_{B B}}{w_{B A}} N_{B}^{2}-2 N_{A} N_{B}>0$. 
Using the definitions of $w_{x y}$ and $\beta_{x}(x, y=A, B)$ we can derive the following:

$$
\begin{aligned}
& w_{A A}-w_{A B} \\
& =(g-f)\left\{\begin{array}{c}
\left(w_{1}-w_{2}\right)\left(\rho_{A}(\alpha) g+\rho_{B}(\alpha) f\right) \\
+\left(w_{2}-w_{3}\right)\left(\rho_{A}(\alpha)(1-g)+\rho_{B}(\alpha)(1-f)\right)
\end{array}\right\}, \\
& w_{B B}-w_{B A} \quad \\
& =(g-f)\left\{\begin{array}{c}
\left(w_{5}-w_{4}\right)\left(\rho_{A}(\alpha) g+\rho_{B}(\alpha) f\right) \\
+\left(w_{6}-w_{5}\right)\left(\rho_{A}(\alpha)(1-g)+\rho_{B}(\alpha)(1-f)\right)
\end{array}\right\} .
\end{aligned}
$$

In Appendix B it is proved that in polymorphic populations $g>f$. When the viabilities satisfy the inequalities in (9), the right-hand sides of both equations (18) are non-negative. Hence, $w_{A A}>w_{A B}$ and $w_{B B}>w_{B A}$. This implies that in polymorphic resident populations inequality (17) is satisfied for all $N_{A}$ and $N_{B}$.

It remains to be examined whether inequality (17) holds for monomorphic equilibria, where $g=f=0$ or 1. From (18) we see that in those cases $w_{A A}=w_{A B}$ and $w_{B B}=w_{B A}$. Thus, (17) is satisfied when $N_{A} \neq N_{B}$. When $N_{A}=N_{B}$ the left hand side of (17) is zero, and thus there is no selection on $p$.

When the density regulation is the same in both patches, however, $N_{A}=N_{B}$ implies that $Q_{A}=Q_{B}$, and from (16) it can be seen that in a non-trivial equilibrium $Q_{A}=1 / w_{A A}$ and $Q_{B}=1 / w_{B B}$. Hence, this can only occur when $w_{3}=w_{6}$ and $g=f=0$, or when $w_{1}=w_{4}$ and $g=f=1$. It turns out that both these equilibria are unstable. This is proven for the case $g=f=0$ and $w_{3}=$ $w_{6}$ in Appendix C. The proof for the other case is completely analogous. Therefore, this situation cannot occur.

When the forms of the functions $Q_{A}$ and $Q_{B}$ differ this may no longer be true. It would have to be examined for specific models whether monomorphic equilibria with $N_{A}=N_{B}$ can be stable. If so, there is no selection on $p$ for such resident populations. However, note that this is an exceptional situation. Therefore, in general, selection will cause $p$ to decrease in monomorphic as well as polymorphic resident populations regardless of the value of $\alpha$.

\subsection{Selection on $\alpha$}

We will now examine the direction of selection on $\alpha$. First note from (5) that whenever $g=f, \beta_{A}$ and $\beta_{B}$ do not depend on $\alpha$. Therefore, there is no selection on $\alpha$ in monomorphic resident populations.

From the previous results it follows that whatever the initial conditions, in the long run $p$ will evolve to zero. Therefore, for polymorphic populations we only need to study the direction of selection on $\alpha$ for $p_{r}=0$. In that case the recurrence equations for the densities of a rare mutant are given by

$$
N_{A}^{\prime}=Q_{A} w_{A A} N_{A}, N_{B}^{\prime}=Q_{B} w_{B B} N_{B},
$$

where the $w_{x x}$ depend on the mating preference of the mutant, $\alpha_{m}$, and the values of $Q_{x}(x=A, B)$ depend on the mating preference of the resident population, $\alpha_{r}$. The growth rate of the mutant population equals the maximum of $Q_{A} w_{A A}$ and $Q_{B} w_{B B}$. First consider $\lambda=$ $Q_{A} w_{A A}$. In that case,

$$
\begin{aligned}
\frac{\partial \lambda}{\partial \alpha_{m}}= & Q_{A} \frac{\partial \beta_{A}}{\partial \alpha_{m}}\left\{\left(w_{1}-w_{2}\right) g\right. \\
& \left.+\left(w_{2}-w_{3}\right)(1-g)\right\},
\end{aligned}
$$

and from (5)

$\frac{\partial \beta_{A}}{\partial \alpha_{m}}=\frac{\partial \rho_{A}(\alpha)}{\partial \alpha_{m}}(g-f)$.

Since $\rho_{A}(\alpha)$ increases in $\alpha$ and $w_{1}>w_{2}>w_{3}, \partial \lambda / \partial \alpha_{m}$ is larger than zero when $g$ is larger than $f$. In a similar way this can be proven for $\lambda=Q_{B} w_{B B}$. As we know that $g>f$ for all $\alpha_{r}$ in polymorphic populations (see Appendix B), this means that there is selection in favour of an increase in mating preference at $p_{r}=0$ in polymorphic populations.

\subsection{Example}

From the previous results we can conclude that in the long run, regardless of the initial resident population, habitat preference will become absolute $(p=0)$ and if the population is polymorphic at that point, mate preference will increase, which will eventually lead to complete reproductive isolation of the populations in the two habitats. Thus, a necessary condition for speciation is that both alleles can coexist at $p=0$ and high values of $\alpha$.

\subsubsection{Stability of monomorphic equilibria}

The possibility of speciation can be studied by looking at the stability conditions of monomorphic equilibria at $p=0$. As an example, we will consider the model that was examined by Beltman et al. (2004), with BevertonHolt density dependence (see Eq. (3)) and mating preference functions as in Eq. (2).

At $p=0$ the recurrence equations for $N_{A}$ and $N_{B}$ are as given in (19), and for $g$ and $f$ we find from (7)

$g^{\prime}=\frac{w_{A A, 1}}{w_{A A}}, \quad f^{\prime}=\frac{w_{B B, 1}}{w_{B B}}$

An equilibrium is stable when the Jacobian of the system of recurrence equations for $N_{A}$ and $N_{B}$ as well as that of the recurrence equations for $g$ and $f$ both have eigenvalues that are smaller than one in absolute value.

There are four types of genetically monomorphic equilibria: two where only one of the habitats is 
occupied, and two where both habitats are occupied. We first consider the situation where only allele $g_{A}$ occurs and only habitat $A$ is occupied. In Appendix D.1 it is shown that, when the viabilities satisfy (9), the equilibrium is stable if

$w_{1}>1 / E, \quad w_{4}<1 / E, \quad w_{5}<2 w_{4}$.

From symmetry considerations it follows that the equilibrium with only habitat $B$ occupied and allele $g_{B}$ present is stable if

$w_{6}>1 / E, \quad w_{3}<1 / E, \quad w_{2}<2 w_{3}$.

Note that these conditions do not depend on $\alpha$.

Next we consider the situation where both habitats are occupied (but the population is genetically monomorphic). In Appendix D.2 it is shown that, when $p=0$, and the viabilities satisfy the conditions in (9), monomorphic equilibria where both habitats are occupied become unstable when $\alpha$ is sufficiently large.

When initially mating with respect to the cultural trait is random $(\alpha=1)$, a monomorphic equilibrium with only allele $g_{A}$ and both habitats occupied is stable when (Appendix D.2)

$$
\begin{aligned}
w_{1} & >1 / E, \\
w_{4} & >1 / E, \\
8 w_{1} w_{4}>3\left(w_{2} w_{4}+w_{1} w_{5}\right) & \\
+ & \sqrt{9\left(w_{2}^{2} w_{4}^{2}+w_{1}^{2} w_{5}^{2}\right)-16 w_{1} w_{2} w_{4} w_{5} .}
\end{aligned}
$$

From symmetry considerations it follows that a monomorphic equilibrium with only allele $g_{B}$ and both habitats occupied is stable when

$$
\begin{aligned}
w_{6}>1 / E, \\
w_{3}>1 / E, \\
8 w_{6} w_{3}>3\left(w_{5} w_{3}+w_{6} w_{2}\right) \\
+\sqrt{9\left(w_{5}^{2} w_{3}^{2}+w_{6}^{2} w_{2}^{2}\right)-16 w_{6} w_{5} w_{3} w_{2} .}
\end{aligned}
$$

When all monomorphic equilibria are unstable at $p=$ 0 , a polymorphic equilibrium will be attained (since fixed points are the only possible types of attractors) after which the mating preference will increase, which leads to speciation. Whether speciation is guaranteed to occur can thus be derived from conditions (23) to (26). For instance, when $w_{4}<1 / E$ and $w_{3}<1 / E$, sufficient conditions for speciation are $w_{5}>2 w_{4}$ and $w_{2}>2 w_{3}$.

\subsubsection{Conditions for speciation}

The conditions for speciation to occur are in reality less strict than those derived from (23) to (26). This is because it is not necessary that all monomorphic equilibria are unstable for a polymorphic equilibrium to be stable. Even when both a monomorphic equilibrium with allele $g_{A}$ present and one with allele $g_{B}$ present are stable, there can in addition be a stable polymorphic equilibrium. The evolutionary outcome then depends on the initial situation.

Fig. 1 illustrates different qualitative outcomes from numerical studies, starting at $p=0.5$ (no habitat preference) and $\alpha=1$ (random mating). If monomorphic equilibria are unstable at this point (as in Fig. 1a), a polymorphic equilibrium will be attained. Evolution will then proceed to lower $p$ and higher $\alpha$, even when a stable monomorphic equilibrium exists (in the black region in Fig. 1a both a monomorphic equilibrium with allele $g_{A}$ present and a polymorphic equilibrium are stable).

An alternative scenario is shown in Fig. 1b. Here, at the initial point $p=0.5$ and $\alpha=1$ a monomorphic equilibrium with allele $g_{A}$ present is stable. When we start in this equilibrium, evolution proceeds to lower $p$, but there is no change in $\alpha$. This leads to instability of the monomorphic equilibria, after which a polymorphic equilibrium is attained, and speciation subsequently occurs.

Speciation does not occur when the system starts in a monomorphic equilibrium, and this equilibrium remains stable on the entire line $\alpha=1$ (Fig. 1c). Numerical analysis showed that this situation is relatively rare, and occurs mainly when female fertility $E$ is low or the difference between $w_{2}$ and $w_{3}$ and between $w_{4}$ and $w_{5}$ is (a)

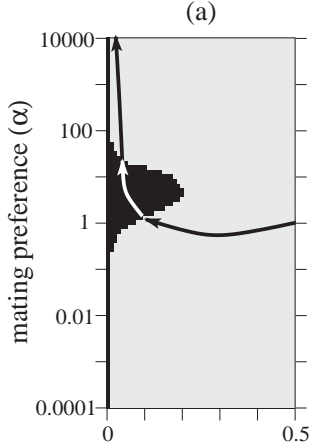

(b)

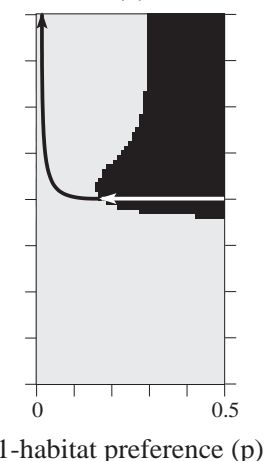

(c)

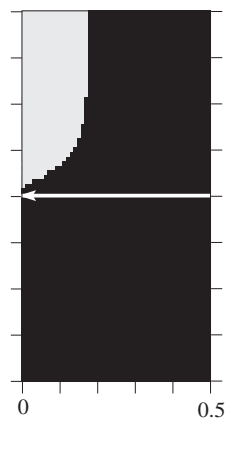

Fig. 1. Prediction of evolutionary trajectories for three qualitatively different parameter settings. In the grey regions a polymorphic equilibrium will be attained because monomorphic equilibria are unstable. In the black region the monomorphic equilibrium with allele $g_{A}$ present is stable, but a polymorphic equilibrium may be stable as well. Trajectories are shown for the case that the location where young are produced and mating are initially random $(p=0.5$ and $\alpha=1)$. In (a), a polymorphic equilibrium is immediately attained. In a part of the trajectory a monomorphic equilibrium is stable, but speciation occurs because the polymorphic equilibrium is also stable. In (b) and (c), evolution proceeds to lower $p$ but there is no evolutionary change in $\alpha$ as long as the resident population is in a monomorphic equilibrium. In (b) the decrease of $p$ causes a secondary switch toward the polymorphic equilibrium, after which completion of speciation follows. In (c) the equilibrium switch does not happen, and therefore mating with respect to the cultural trait remains random. Parameters: $K=0.001, w_{1}=1.0$, $w_{6}=1.0$, and in (a) $E=2, w_{2}=0.8, w_{3}=0.2, w_{4}=0.2, w_{5}=0.8$, in (b) $E=10, w_{2}=0.4, w_{3}=0.0, w_{4}=0.2, w_{5}=0.6$, and in (c) $E=10$, $w_{2}=0.4, w_{3}=0.2, w_{4}=0.6, w_{5}=0.8$. 
small. In such cases, evolution will not lead to speciation because genetic diversification does not take place. Instead, although all individuals produce their young exclusively in one of the two habitats, mating with respect to the cultural trait is random. Hence, a clear separation in habitat exploitation of individuals takes place, but not speciation.

\subsubsection{Individual-based model}

To check the validity of the predicted evolutionary trajectories, we used an individual-based simulation model based on similar assumptions as the recurrence equations (a program written in $\mathrm{C}$, available on request). We consider the situation that the population initially consists of females and males of type $c_{A} g_{A} g_{A}$ with $\alpha=1$ and $p=0.5$. Mating, young production, density regulation and viability selection are implemented according to the previously described life history. As in the previous model, population size is not fixed over time but depends on density regulation and viability selection. The traits $p$ and $\alpha$ are inherited from both parents, using additive genetics (note that males carry but do not express these traits). Newborns can have mutations in three characteristics: (i) the probability of egg laying mistakes $p$, (ii) the strength of the mating preference $\alpha$, and (iii) the genetic trait that determines viability $\left(g_{A} g_{A}, g_{A} g_{B}\right.$, or $\left.g_{B} g_{B}\right)$. Mutations in the three traits are independent and occur each with a constant probability per generation $\mu$. For mutations in $p$ or $\alpha$, the mutation step is drawn randomly from a normal distribution with average 0 and standard deviation $\sigma$. To avoid biases in the step size of mutations in a certain direction, we transform $p$ and $\alpha$ to a linear scale that varies from $-\infty$ to $\infty$ before adding mutations (e.g., in the case of $\alpha$ this means taking the logarithm of the $\alpha$ value of an individual). Subsequently, the mutation is added and the resulting values are transformed back to their original scale. We apply the same procedure to determine the trait of a young: first the trait values of the two parents are transformed to the linear scale, subsequently their average is taken, and the resulting value is transformed back.

The mathematical analysis showed that, during the evolution of $p$ and $\alpha$, the population can switch from a boundary equilibrium that contains only one type of allele, to an internal equilibrium that contains both types of alleles (e.g., Fig. 1b). To allow for this in the individual-based model, we include mutations in the genetic trait that determines viability. This is incorporated as a homozygote $\left(g_{A} g_{A}\right.$ or $\left.g_{B} g_{B}\right)$ mutating into a heterozygote $\left(g_{A} g_{B}\right)$, or vice versa. This results in a constant, low influx of new mutants in the viability gene, allowing for the process of genetic divergence to occur at different stages of evolution.

Using these simulations we studied the evolution of the three traits over a large number of generations. We did not examine the parameter space exhaustively. Instead, we performed simulations for several parameter settings for which the mathematical analysis predicted qualitatively different results. As expected, the switching between different equilibria is not as clear cut as in the deterministic model, and over evolutionary time there is quite some variation in the evolving traits. Nevertheless, the predictions of the mathematical analysis were confirmed by the individual-based analogue. A typical simulation is shown in Fig. 2: In this example the monomorphic equilibrium with allele $g_{A}$ present is stable at the initial point ( $p=0.5$ and $\alpha=1)$. There is selection toward lower $p$ (generation 0 to approximately $50,000)$, and this modifies the stability of the monomorphic equilibrium. As a result, a polymorphic equilibrium is attained. Subsequently, a decrease in genetic mixing leads to completion of the speciation process (generation 50,000-1,000,000).
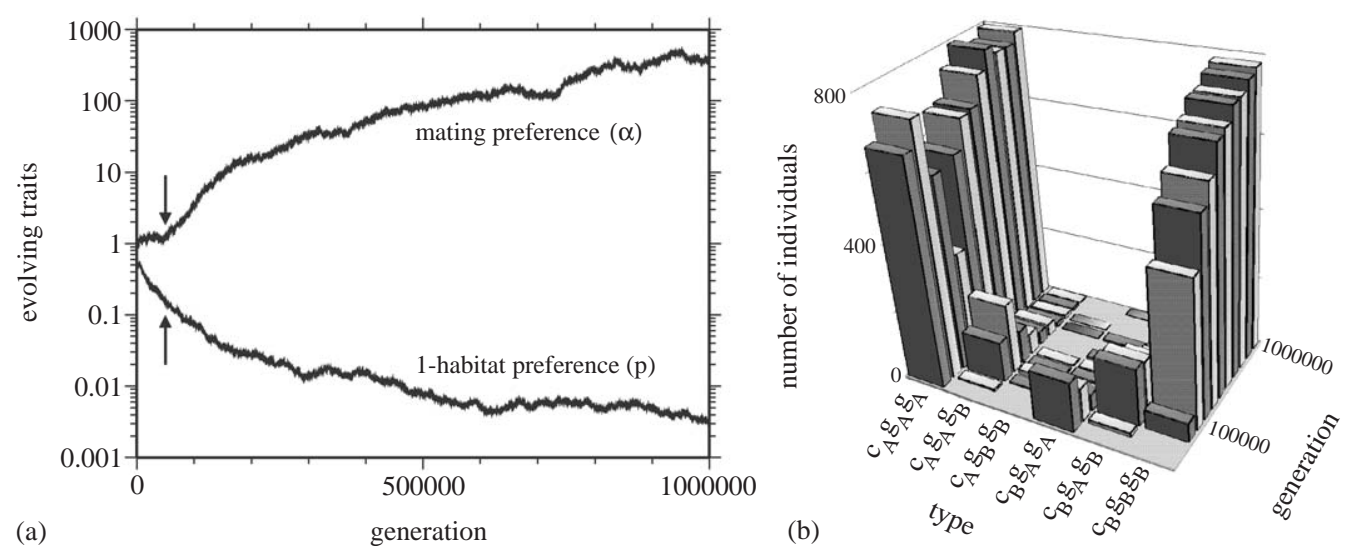

Fig. 2. (a) Evolution of habitat and mating preference in the individual-based simulation model, starting from random mating $(\alpha=1)$ and random young production $(p=0.5$ ). (b) The population constitution in the course of evolution. Initially the system is in a monomorphic allele $A$ equilibrium and cannot attain the polymorphic equilibrium. Evolution then proceeds to lower $p$ (there is no selection on $\alpha$ yet). After approximately 50,000 generations (denoted by the arrows in (a)), the polymorphic equilibrium is attained. Genetic mixing then further decreases due to evolution to lower $p$ and higher $\alpha$. Parameters: $E=10, w_{2}=0.6, w_{3}=0.2, w_{4}=0.2, w_{5}=0.6, \mu=0.001, \sigma=0.25, K=0.0001$. 


\section{Discussion}

We investigated a mechanism that is often ignored in the literature, speciation through the learning of habitat features. When habitat imprinting causes both habitatdependent assortative mating and a tendency to produce young in this habitat, this may lead to speciation in three steps: (i) colonization of a new habitat, (ii) genetic divergence of the two groups by adaptation to the habitats, and (iii) a decrease of genetic mixing between the lineages. We have previously focussed on the first two steps of this speciation mechanism by analysing a gene-culture coevolutionary model (Beltman et al., 2004). Here we took a more general approach, and investigated whether there is selection in favour of decreased genetic mixing. We showed that for a large class of models there is selection toward producing young more frequently in the habitat that individuals exploit themselves. In addition, once habitat preference is strong and genetic divergence has occurred, there is selection toward stronger habitat-dependent assortative mating due to hybrid inferiority. Speciation is thus guaranteed when genetic divergence occurs at strong habitat preferences. Sufficient conditions for speciation in a particular model can therefore be derived from the stability of genetically monomorphic equilibria at strong habitat preferences. We derived these conditions for the example studied in Beltman et al. (2004). We also demonstrated that the conditions for speciation are in reality less strict because stability of monomorphic equilibria does not imply that polymorphic equilibria are unstable. When polymorphic as well as monomorphic equilibria are stable, the occurrence of speciation depends on the initial situation. For instance, genetic divergence may not occur initially, but evolutionary changes in habitat preference may render a monomorphic equilibrium unstable. In that case, genetic diversification followed by speciation will occur secondarily (e.g., Fig. 1b). Finally, we investigated an individual-based analogue of the model of Beltman et al. (2004), and confirmed the occurrence of speciation predicted by the mathematical analysis.

The models for migration modification, studied by Karlin and McGregor (1972), Balkau and Feldman (1973), and Karlin and McGregor (1974), are closely related to ours. However, there it is assumed that mating preference is absolute, i.e. that $\alpha$ is infinitely large. Speciation is then complete when the migration fraction is zero (or, in our terminology the habitat preference equals one). Furthermore, they do not consider the dynamics of the population densities but assume that these have positive equilibrium values. They showed that for several combinations of viability selection in the two patches, including the ones we consider, there is selection towards decreased migration. Our results generalize theirs in two respects: First, we showed that if the viabilities satisfy the inequalities in (9) selection towards decreased migration occurs regardless of the value of the mating preference; Second, we explicitly considered a wide variety of models for local density dependence and allowed for the possibility of local extinction. Furthermore, we derived conditions for completion of reproductive isolation through selection towards increased mating preferences.

The decrease of genetic mixing due to hybrid inferiority was originally hypothesized by Dobzhansky (1940) and the process has been termed reinforcement by Blair (1955). It was introduced in the context of geographical speciation: an initial allopatric phase during which genetic differences accumulate is followed by a sympatric phase, during which reinforcement results in reproductive character displacement. According to several theoretical studies, reinforcement of prezygotic isolation is possible under certain conditions (e.g., Liou and Price, 1994; Servedio and Kirkpatrick, 1997; Servedio, 2000; Kirkpatrick, 2001; Sadedin and Littlejohn, 2003; Servedio and Sætre, 2003). Most importantly, it was found that a high hybrid viability and high recombination between alleles affecting mate choice and those affecting hybrid viability oppose reinforcement (Felsenstein, 1981; Trickett and Butlin, 1994; Servedio, 2000). The final step of the speciation mechanism we study is similar to the reinforcement process, although it operates in a different context. Here, the decrease of genetic mixing appears to lack the relatively stringent conditions under which reinforcement is expected to occur. Probably the crucial factor causing this difference in results is the absence of the opposing effect of recombination: Because learning instead of a genetic mechanism determines which of the habitats is preferred, recombination cannot destroy the association between alleles that determine viability and the learned preference. Therefore, our results do not contradict the predictions of previous models that recombination opposes reinforcement (Felsenstein, 1981; Trickett and Butlin, 1994; Servedio, 2000)

We assumed that the trait that determines viability is based on only one locus. It is known that the number of loci for an ecological trait can influence the probability of sympatric speciation (e.g., Kondrashov, 1986). We think that an increase in the number of viability loci in our model may indeed make the step of genetic diversification (studied in Beltman et al., 2004) more difficult, because recombination between these multiple loci may destroy associations between them. However, once genetic diversification has been achieved, the reinforcement step is likely to occur without problem because recombination again forms no obstacle there (see above). However, the presence of multiple viability loci is an interesting extension of the model and remains to be investigated. 
We assumed that female mate choice is not costly. It would probably be most realistic to make the cost of female choosiness dependent on the frequency of the preferred males (as is for example the case in Gavrilets and Boake, 1998; Beltman et al., 2003). We expect that this assumption would not dramatically influence the reinforcement step, because, once the population has reached the polymorphic equilibrium, males of both cultural traits are abundant (see for example Fig. 2b). However, as we already discussed in Beltman et al. (2004), the step of genetic diversification is likely to become more difficult when mate choice is costly, because at that time the preferred males may not yet be present in high frequencies.

The learning of habitat features occurs in many species. In the case of the brood-parasitic finches the learning of foster species' songs causes a "true" mating and egg laying preference. In most other cases these will be a by-product of feeding preference (e.g., in phytophagous insects). Our assumption that the preference to produce young in a particular habitat and the mating preference are independent traits is then violated. Instead, in such cases there is a single underlying trait that causes the two effects. It is not likely that this would prevent speciation, because as we showed selection on the two effects usually operates in concert toward speciation. Although we did not test this, we thus expect that such a coupling would speed up rather than hamper the speciation process.

We have studied speciation under the assumption that the learning of habitat features is present from the start. However, we assumed that the effect that such learning has on mating behaviour and location where young are produced can change in the course of evolution. Indeed, we included a scenario where there is initially no effect of learning on mating and location where young are produced (this is the case at $p=0.5$ and $\alpha=1$ ). The evolutionary origin of the learning of habitat features, however, is not yet completely understood. It may have evolved for reasons unrelated to the speciation mechanism we study or, alternatively, the evolution of learning and subsequent speciation may be due to adaptation to the environment. For example, brood-parasitic finches use their foster species' songs for mate choice and host searching. Probably, their ancestors were generalist brood parasites and may not have learned the foster species' songs (for a discussion on this topic see Beltman et al., 2003). The learning and use of these songs may have evolved in response to counteradaptations of the hosts against brood parasitism. Learning their songs helps to specialize on the parasitization of a particular foster species. Among brood parasites there is no other group than the Viduidae where the copying of foster species' song occurs (Davies (2000)). However, brownheaded cowbirds are able to discriminate between songs of different host species (Hauber et al. (2002)), although they are generalist brood parasites. Possibly, the abovedescribed process may occur in this group as well, leading to the specialization of cowbirds' descendants on particular hosts and maybe also to the copying of their songs.

Previous models of speciation have usually ignored the possible role of learning processes, but instead focused on the geographical mode of speciation (see references in Introduction). However, as we discussed previously (Beltman et al., 2004) the learning of habitat features provides an equally powerful, analogous mechanism of generating assortative mating as geographical separation. The possible importance of the learning of habitat features on speciation has been hinted at by several researchers (e.g., Thorpe, 1945; Maynard Smith, 1966; Rice, 1984; Kondrashov and Mina, 1986; West-Eberhard, 2003), but has received relatively little attention so far. The present study (in combination with Beltman et al., 2004) demonstrates the effectiveness of this speciation mechanism theoretically. We think that a stronger experimental and theoretical focus on the role of learning in speciation has much potential for increasing our understanding of the evolution of new species.

\section{Acknowledgments}

We thank C. Rueffler for advice on adaptive dynamics, and C. ten Cate, M. Durinx, P. Edelaar, A.S. Kondrashov, K.N. Laland, J.A.J. Metz, K. Riebel, C. Rueffler, H. Slabbekoorn, and two anonymous referees for providing useful suggestions on previous versions of this manuscript. This study was supported by the Research Council for Earth and Life Sciences (ALW), which is subsidized by the Netherlands Organization for Scientific Research (NWO).

\section{Appendix A. Derivation of recurrence relations (6) and (7)}

For notational convenience we define the following matrices:

$$
W_{A}=\left(\begin{array}{ccc}
w_{1} & 0 & 0 \\
0 & w_{2} & 0 \\
0 & 0 & w_{3}
\end{array}\right), \quad W_{B}=\left(\begin{array}{ccc}
w_{4} & 0 & 0 \\
0 & w_{5} & 0 \\
0 & 0 & w_{6}
\end{array}\right)
$$

and

$$
M_{A}=\left(\begin{array}{ccc}
\beta_{A} & \frac{1}{2} \beta_{A} & 0 \\
\left(1-\beta_{A}\right) & \frac{1}{2} & \beta_{A} \\
0 & \frac{1}{2}\left(1-\beta_{A}\right) & \left(1-\beta_{A}\right)
\end{array}\right) .
$$


The matrix $M_{B}$ is the same as $M_{A}$, but with $\beta_{B}$ substituted for $\beta_{A}$. If we denote the type densities by a vector $N=\left(N_{1}, \ldots, N_{6}\right)^{T}$ (as in Table 1), the population dynamics are described by

$N^{\prime}=M N$,

where $M$ is a $6 \times 6$ matrix with the following structure:

$M=\left(\begin{array}{cc}(1-p) Q_{A} W_{A} M_{A} & p Q_{A} W_{A} M_{B} \\ p Q_{B} W_{B} M_{A} & (1-p) Q_{B} W_{B} M_{B}\end{array}\right)$.

Note that the equations given in the Appendix of Beltman et al. (2004) can be cast in this form too, with the definitions of $\rho_{x}(\alpha)$ and $Q_{x}$ as in Eqs. (2) and (3).

The dimension of the state space can be reduced by considering the following state variables in stead of type densities:

$H_{1}=N_{1}+\frac{1}{2} N_{2}, \quad H_{2}=N_{3}+\frac{1}{2} N_{2}$,

$H_{3}=N_{4}+\frac{1}{2} N_{5}, \quad H_{4}=N_{6}+\frac{1}{2} N_{5}$,

for example $2 H_{1}$ is the density of allele $g_{A}$ in habitat $A$.

We will now show that

$H^{\prime}=R H$,

where $H$ is the vector $\left(H_{1}, \ldots, H_{4}\right)^{T}$, and

$R=\left(\begin{array}{cc}(1-p) Q_{A} R_{A A} & p Q_{A} R_{A B} \\ p Q_{B} R_{B A} & (1-p) Q_{B} R_{B B}\end{array}\right)$.

Here,

$R_{A A}=\left(\begin{array}{cc}w_{1} \beta_{A}+\frac{1}{2} w_{2}\left(1-\beta_{A}\right) & \frac{1}{2} w_{2} \beta_{A} \\ \frac{1}{2} w_{2}\left(1-\beta_{A}\right) & \frac{1}{2} w_{2} \beta_{A}+w_{3}\left(1-\beta_{A}\right)\end{array}\right)$.

In $R_{A B}, \beta_{A}$ is substituted by $\beta_{A}$ in this matrix. In $R_{B A}$ and $R_{B B}, w_{1}, w_{2}$ and $w_{3}$ are replaced by respectively $w_{4}$, $w_{5}$ and $w_{6}$, and in $R_{B B}$, moreover, $\beta_{B}$ is substituted for $\beta_{A}$.

Let $H_{A}$ be the vector $\left(H_{1}, H_{2}\right)^{T}, H_{B}=\left(H_{3}, H_{4}\right)^{T}$, and $F_{A}=\left(N_{1}, N_{2}, N_{3}\right)^{T}, F_{B}=\left(N_{4}, N_{5}, N_{6}\right)^{T}$ then $H_{A}=$ $X F_{A}$, and $H_{B}=X F_{B}$ with

$X=\left(\begin{array}{ccc}1 & \frac{1}{2} & 0 \\ 0 & \frac{1}{2} & 1\end{array}\right)$.

From (A.4) it follows that

$H_{A}^{\prime}=(1-p) Q_{A} X W_{A} M_{A} F_{A}+p Q_{A} X W_{A} M_{B} F_{B}$.

It is easily verified that

$X W_{A} M_{A}=R_{A A} X \quad$ and $\quad X W_{A} M_{B}=R_{A B} X$,

which implies that

$H_{A}^{\prime}=(1-p) Q_{A} R_{A A} H_{A}+p Q_{A} R_{A B} H_{B}$.
The relation

$H_{B}^{\prime}=p Q_{B} R_{B A} H_{A}+(1-p) Q_{B} R_{B B} H_{B}$

is derived analogously.

The relations in (6) can be derived by noting that

$N_{A}=H_{1}+H_{2}=H_{A}^{T}\left(\begin{array}{l}1 \\ 1\end{array}\right)$

and similarly $\mathrm{N}_{B}=\mathrm{H}_{3}+\mathrm{H}_{4}$. The recurrence equations for $g$ and $f$ follow from $g^{\prime}=H_{1}^{\prime} / N_{A}^{\prime}$ and $f^{\prime}=H_{3}^{\prime} / N_{B}^{\prime}$.

\section{Appendix B. Proof that $g \geqslant f$ with equality only when $g=$ 0 or 1}

From the recurrence equation for $g$ in (7) and (16) it follows that at equilibrium

$(1-p) N_{A}\left\{w_{A A, 1}-g w_{A A}\right\}+p N_{B}\left\{w_{A B, 1}-g w_{A B}\right\}=0$.

Further,

$$
\begin{aligned}
w_{A A, 1}-g w_{A A}= & \beta_{A}\left\{\frac{1}{2} w_{2}+g(1-g)\left(\left(w_{1}-w_{2}\right)\right.\right. \\
& \left.\left.-\left(w_{2}-w_{3}\right)\right)\right\} \\
& +\left(w_{2}-w_{3}\right) g(1-g)-\frac{1}{2} w_{2} g, \\
w_{A B, 1}-g w_{A B}= & \beta_{B}\left\{\frac{1}{2} w_{2}+f(1-g)\left(w_{1}-w_{2}\right)\right. \\
& \left.-g(1-f)\left(w_{2}-w_{3}\right)\right\} \\
& +w_{2} f(1-g)-w_{3} g(1-f)-\frac{1}{2} w_{2} f .
\end{aligned}
$$

We will now show that if $f>g$ both these expressions are positive, which contradicts (B.1). First note that $\frac{1}{2} w_{2}+$ $g(1-g)\left(\left(w_{1}-w_{2}\right)-\left(w_{2}-w_{3}\right)\right)$ is positive. This is obviously true when $\left(w_{1}-w_{2}\right)>\left(w_{2}-w_{3}\right)$. When $\left(w_{1}-\right.$ $\left.w_{2}\right)<\left(w_{2}-w_{3}\right)$ this function has a global minimum at $g=\frac{1}{2}$, and obviously its value is larger than zero at this point. If $f>g$ then the multiplication factor for $\beta_{B}$ in the second equation above is larger than this value, so that value must then be positive too. From (5) it further follows that in this case $\beta_{A}$ and $\beta_{B}$ are both larger than $g$. Therefore,

$$
\begin{aligned}
w_{A A, 1}-g w_{A A}> & g\left\{\frac{1}{2} w_{2}+g(1-g)\left(\left(w_{1}-w_{2}\right)\right.\right. \\
& \left.\left.-\left(w_{2}-w_{3}\right)\right)\right\} \\
& +\left(w_{2}-w_{3}\right) g(1-g)-\frac{1}{2} w_{2} g \\
= & g(1-g)\left\{g\left(w_{1}-w_{2}\right)+(1-g)\right. \\
& \left.\times\left(w_{2}-w_{3}\right)\right\}>0,
\end{aligned}
$$


and

$$
\begin{aligned}
w_{A B, 1}-g w_{A B}> & g\left\{\frac{1}{2} w_{2}+f(1-g)\left(w_{1}-w_{2}\right)\right. \\
& \left.-g(1-f)\left(w_{2}-w_{3}\right)\right\} \\
& +w_{2} f(1-g)-w_{3} g(1-f)-\frac{1}{2} w_{2} f \\
= & f\left\{\begin{array}{c}
g(1-g)\left(w_{1}-w_{2}\right)+g^{2}\left(w_{2}-w_{3}\right) \\
+w_{2}(1-g)+w_{3} g-\frac{1}{2} w_{2}
\end{array}\right\} \\
& -\frac{1}{2} w_{2} g \\
= & f\left\{g(1-g)\left(\left(w_{1}-w_{2}\right)+\left(w_{2}-w_{3}\right)\right)\right. \\
& \left.+\frac{1}{2} w_{2}\right\}-\frac{1}{2} w_{2} g \\
> & g\left\{g ( 1 - g ) \left(\left(w_{1}-w_{2}\right)\right.\right. \\
& \left.\left.+\left(w_{2}-w_{3}\right)\right)\right\}>0 .
\end{aligned}
$$

Thus, if $f>g$ both expressions on the left-hand side of (B.1) are positive, which cannot be true. Therefore $f$ must be smaller than or equal to $g$.

When $g$ is equal to $f$, it can be seen from (5) that $\beta_{A}=\beta_{B}=g$. And from (B.1) and (B.2) we find

$$
\begin{gathered}
g\left\{g(1-g)\left(\left(w_{1}-w_{2}\right)-\left(w_{2}-w_{3}\right)\right)\right\} \\
+\left(w_{2}-w_{3}\right) g(1-g)=0,
\end{gathered}
$$

with solutions $g=0$ or 1 . The other solution must satisfy

$g\left\{\left(\left(w_{1}-w_{2}\right)-\left(w_{2}-w_{3}\right)\right)\right\}+\left(w_{2}-w_{3}\right)=0$,

and so

$g=\frac{\left(w_{2}-w_{3}\right)}{\left(w_{2}-w_{3}\right)-\left(w_{1}-w_{2}\right)}$.

However, there is no $g$ between 0 and 1 that satisfies this equation, which means that there are no polymorphic equilibria with $g$ equal to $f$.

\section{Appendix C. Proof that when $w_{3}=w_{6}$ the equilibrium with $g=f=0 \wedge N_{A}=N_{B}$ is unstable}

A necessary condition for stability of this equilibrium is that the absolute values of all eigenvalues of the Jacobian of the recurrence equations for $N_{A}$ and $N_{B}$ in (6) are smaller than one. The partial derivatives are

$$
\left(\begin{array}{ll}
\frac{\partial Q_{A}}{\partial N_{A}}\left((1-p) w_{A A} N_{A}+p w_{A B} N_{B}\right) & \frac{\partial Q_{A}}{\partial N_{B}}\left((1-p) w_{A A} N_{A}+p w_{A B} N_{B}\right) \\
+(1-p) Q_{A} w_{A A} & +p Q_{A} w_{A B} \\
\frac{\partial Q_{B}}{\partial N_{A}}\left(p w_{B A} N_{A}+(1-p) w_{B B} N_{B}\right) & \frac{\partial Q_{B}}{\partial N_{B}}\left(p w_{B A} N_{A}+(1-p) w_{B B} N_{B}\right) \\
+p Q_{B} w_{B A} & +(1-p) Q_{B} w_{B B}
\end{array}\right)
$$

In the considered case

$w_{A A}=w_{A B}=w_{B B}=w_{B A}=w$, and when $N_{A}=N_{B}=N$ the equilibrium conditions lead to

$Q_{A} w_{A A}=Q_{B} w_{B B}=1$.

Furthermore, when density regulation is similar in the two habitats we know that at the equilibrium where $N_{A}=N_{B}, \quad \partial Q_{A} / \partial N_{A}=\partial Q_{B} / \partial N_{B} \quad$ and $\quad \partial Q_{A} / \partial N_{B}=$ $\partial Q_{B} / \partial N_{A}$. Denote these values by respectively $\gamma$ and $\eta$. The Jacobian then becomes

$$
\left(\begin{array}{cc}
\gamma w N+(1-p) & \eta w N+p \\
\eta w N+p & \gamma w N+(1-p)
\end{array}\right)
$$

It is easily shown that the largest eigenvalue of this matrix is larger than one, which implies that the equilibrium is unstable.

\section{Appendix D. Stability conditions for monomorphic equilibria at $p=0$}

From (3) it can be derived that when $p=0$ :

$\frac{\partial Q_{A}}{\partial N_{B}}=\frac{\partial Q_{B}}{\partial N_{A}}=0, \quad \frac{\partial Q_{x}}{\partial N_{x}}=-K Q_{x}^{2}(x=A, B)$.

Hence, the matrix of partial derivatives for the system $\left\{N_{A}, N_{B}\right\}$ equals (see (C.1))

$$
\left(\begin{array}{cc}
-K Q_{A}^{2} w_{A A} N_{A}+Q_{A} w_{A A} & 0 \\
0 & -K Q_{B}^{2} w_{B B} N_{B}+Q_{B} w_{B B}
\end{array}\right) .
$$

\section{D.1. One habitat occupied}

We consider the equilibrium with $g=1, N_{A}>0$ and $N_{B}=0$. At this equilibrium we see from (2) that $\rho_{A}(\alpha)=$ $1, \rho_{B}(\alpha)=0$, from (5) that $\beta_{A}=\beta_{B}=1$, and from (8) that $w_{A A, 1}=w_{A A}=w_{1}$ and $w_{B B, 1}=w_{B B}=w_{4}$. Filling in $N_{B}=0$ in the second equation in (3) gives $Q_{B}=E$. From the equilibrium condition for $N_{A}$ it follows that $Q_{A}=1 / w_{1}$. Substituting these values in (D.2) gives the Jacobian for $\left\{N_{A}, N_{B}\right\}$ :

$\left(\begin{array}{cc}-K \frac{1}{w_{1}} N_{A}+1 & 0 \\ 0 & E w_{4}\end{array}\right)$.

From $Q_{A}=1 / w_{1}$ we can derive that the equilibrium value of $N_{A}$ equals $\frac{1}{K}\left(w_{1}-\frac{1}{E}\right)$, so the eigenvalues are

$\lambda_{1}=\frac{1}{E w_{1}}, \quad \lambda_{2}=E w_{4}$. 
We now turn to the Jacobian for $g$ and $f$. From (5) we find

$$
\begin{aligned}
& \left(\frac{\partial \beta_{A}}{\partial g}\right)_{g=1, f=0}=\rho_{A}(\alpha)=1, \\
& \left(\frac{\partial \beta_{A}}{\partial f}\right)_{g=1, f=0}=1-\rho_{A}(\alpha)=0, \\
& \left(\frac{\partial \beta_{B}}{\partial g}\right)_{g=1, f=0}=1-\rho_{B}(\alpha)=1, \\
& \left(\frac{\partial \beta_{B}}{\partial f}\right)_{g=1, f=0}=\rho_{B}(\alpha)=0,
\end{aligned}
$$

and from (8)

$$
\begin{aligned}
& \left(\frac{\partial w_{A A, 1}}{\partial g}\right)_{g=1, f=0}=2\left(w_{1}-\frac{1}{2} w_{2}\right), \\
& \left(\frac{\partial w_{A A}}{\partial g}\right)_{g=1, f=0}=2\left(w_{1}-w_{2}\right), \\
& \left(\frac{\partial w_{A A, 1}}{\partial f}\right)_{g=1, f=0}=\left(\frac{\partial w_{A A}}{\partial f}\right)_{g=1, f=0}=0, \\
& \left(\frac{\partial w_{B B, 1}}{\partial g}\right)_{g=1, f=0}=\frac{1}{2} w_{5}, \\
& \left(\frac{\partial w_{B B}}{\partial g}\right)_{g=1, f=0}=w_{5}, \\
& \left(\frac{\partial w_{B B, 1}}{\partial f}\right)_{g=1, f=0}=w_{4}-\frac{1}{2} w_{5}, \\
& \left(\frac{\partial w_{B B}}{\partial g}\right)_{g=1, f=0}=w_{4}-w_{5} .
\end{aligned}
$$

Substituting these values in the partial derivatives of $g^{\prime}$ and $f^{\prime}$ gives the Jacobian:

$$
\left(\begin{array}{cc}
\frac{w_{2}}{w_{1}} & 0 \\
-\frac{1}{2} \frac{w_{5}}{w_{4}} & \frac{1}{2} \frac{w_{5}}{w_{4}}
\end{array}\right)
$$

with eigenvalues

$\lambda_{3}=\frac{w_{2}}{w_{1}}, \quad \lambda_{4}=\frac{1}{2} \frac{w_{5}}{w_{4}}$.

For an equilibrium to be stable, all eigenvalues should be smaller than one. This is true for $\lambda_{3}$ because $w_{2}<w_{1}$. The stability conditions that follow from the other eigenvalues in (D.4) and (D.8) are summarized in (23) of the main text.

\section{D.2. Both habitats occupied}

We consider the equilibrium at which $g=f=1$. At this equilibrium we have: $\beta_{A}=\beta_{B}=1$, and $w_{A A, 1}=$ $w_{A A}=w_{1}, w_{B B, 1}=w_{B B}=w_{4}$. From the equilibrium condition for $N_{A}$ and $N_{B}$ it follows that $Q_{A}=1 / w_{1}$ and $Q_{B}=1 / w_{4}$. From $Q_{A}=1 / w_{1}$ we can derive that the equilibrium value of $N_{A}$ equals $\frac{1}{K}\left(w_{1}-\frac{1}{E}\right)$. Similarly, the equilibrium value of $N_{B}$ is $\frac{1}{K}\left(w_{4}-\frac{1}{E}\right)$. Substituting these values in (D.2) gives the eigenvalues:

$\lambda_{1}=\frac{1}{E w_{1}}, \quad \lambda_{2}=\frac{1}{E w_{4}}$,

which gives the first two conditions in (25).

From (5) we find

$$
\begin{aligned}
& \left(\frac{\partial \beta_{A}}{\partial g}\right)_{g=f=1}=\rho_{A}(\alpha), \\
& \left(\frac{\partial \beta_{A}}{\partial f}\right)_{g=f=1}=1-\rho_{A}(\alpha), \\
& \left(\frac{\partial \beta_{B}}{\partial g}\right)_{g=f=1}=1-\rho_{B}(\alpha), \\
& \left(\frac{\partial \beta_{B}}{\partial f}\right)_{g=f=1}=\rho_{B}(\alpha),
\end{aligned}
$$

and from (8)

$$
\begin{aligned}
\left(\frac{\partial w_{A A, 1}}{\partial g}\right)_{g=f=1} & =\left(w_{1}-\frac{1}{2} w_{2}\right)\left(1+\rho_{A}(\alpha)\right), \\
\left(\frac{\partial w_{A A}}{\partial g}\right)_{g=f=1} & =\left(w_{1}-w_{2}\right)\left(1+\rho_{A}(\alpha)\right), \\
\left(\frac{\partial w_{A A, 1}}{\partial f}\right)_{g=f=1} & =\left(1-\rho_{A}(\alpha)\right)\left(w_{1}-\frac{1}{2} w_{2}\right), \\
\left(\frac{\partial w_{A A}}{\partial f}\right)_{g=f=1} & =\left(1-\rho_{A}(\alpha)\right)\left(w_{1}-w_{2}\right) .
\end{aligned}
$$

Substituting these values in the partial derivatives of $g^{\prime}$ and using symmetry arguments for $f^{\prime}$ we find the Jacobian matrix

$$
\left(\begin{array}{cc}
\left(1+\rho_{A}(\alpha)\right)\left(\frac{1}{2} \frac{w_{2}}{w_{1}}\right) & \left(1-\rho_{A}(\alpha)\right)\left(\frac{1}{2} \frac{w_{2}}{w_{1}}\right) \\
\left(1-\rho_{B}(\alpha)\right)\left(\frac{1}{2} \frac{w_{5}}{w_{4}}\right) & \left(1+\rho_{B}(\alpha)\right)\left(\frac{1}{2} \frac{w_{5}}{w_{4}}\right)
\end{array}\right) .
$$

Both $\rho_{A}(\alpha)$ and $\rho_{B}(\alpha)$ increase monotonically in $\alpha$. These functions equal zero when $\alpha=0$ and tend to one as $\alpha$ becomes infinitely large (see Eq. (2)).

At $\alpha=1$ the function values equal 0.5 , which is of special interest because it represents random mating. When $\alpha$ equals one, the Jacobian becomes

$$
\left(\begin{array}{ll}
\frac{3}{4} \frac{w_{2}}{w_{1}} & \frac{1}{4} \frac{w_{2}}{w_{1}} \\
\frac{1}{4} \frac{w_{5}}{w_{4}} & \frac{3}{4} \frac{w_{5}}{w_{4}}
\end{array}\right),
$$

with eigenvalues

$$
\lambda_{3,4}=\frac{3\left(w_{2} w_{4}+w_{1} w_{5}\right) \pm \sqrt{9\left(w_{2}^{2} w_{4}^{2}+w_{1}^{2} w_{5}^{2}\right)-16 w_{1} w_{2} w_{4} w_{5}}}{8 w_{1} w_{4}} .
$$


The expression under the root is always positive because $w_{1}>w_{2}$ and $w_{5}>w_{4}$. Hence, the eigenvalues are real numbers. The last stability condition in (25) at $p=0$ and $\alpha=1$ then follows from demanding that the eigenvalue with the positive root is smaller than one.

In the limit for $\alpha \rightarrow \infty$ the Jacobian tends to

$$
\left(\begin{array}{cc}
\frac{w_{2}}{w_{1}} & 0 \\
0 & \frac{w_{5}}{w_{4}}
\end{array}\right)
$$

with eigenvalues

$\lambda_{3}=\frac{w_{2}}{w_{1}}, \quad \lambda_{4}=\frac{w_{5}}{w_{4}}$.

Because $w_{2}<w_{1}$ and $w_{5}>w_{4}, \lambda_{3}$ is always smaller than one and $\lambda_{4}$ is always larger than one. Hence, for large values of $\alpha$, the equilibrium will be unstable.

\section{References}

Aoki, K., Feldman, M.W., Kerr, B., 2001. Models of sexual selection on a quantitative genetic trait when preference is acquired by sexual imprinting. Evolution 55, 25-32.

Balkau, B.J., Feldman, M.W., 1973. Selection for migration modification. Genetics 74, 171-174.

Beltman, J.B., Haccou, P., ten Cate, C., 2003. The impact of learning foster species' song on the evolution of specialist avian brood parasitism. Behav. Ecol. 16, 917-923.

Beltman, J.B., Haccou, P., ten Cate, C., 2004. Learning and colonization of new niches: a first step toward speciation. Evolution 58, 35-46.

Blair, W.F., 1955. Mating call and stage of speciation in the Microhyla olivacea-M. carolinensis complex. Evolution 9, 469-480.

Coyne, J.A., Orr, H.A., 2004. Speciation. Sinauer Associates, Inc., Sunderland, MA.

Davies, N.B., 2000. Cuckoos, Cowbirds and other Cheats. T. \& A. D Poyser, London.

Davis, J.M., Stamps, J.A., 2004. The effect of natal experience on habitat preferences. Trends Ecol. Evol. 19, 411-416.

Dieckmann, U., Doebeli, M., 1999. On the origin of species by sympatric speciation. Nature 400, 354-357.

Dieckmann, U., Law, R., 1996. The dynamical theory of coevolution: a derivation from stochastic ecological processes. J. Math. Biol. 34, 579-612.

Dobzhansky, T., 1940. Speciation as a stage in evolutionary divergence. Am. Nat. 74, 312-321.

Ellers, J., Slabbekoorn, H., 2003. Song divergence and male dispersal among bird populations: a spatially explicit model testing the role of vocal learning. Anim. Behav. 65, 671-681.

Felsenstein, J., 1981. Skepticism towards Santa Rosalia, or why are there so few kinds of animals? Evolution 35, 124-138.

Fry, J.D., 2003. Multilocus models of sympatric speciation: Bush versus Rice versus Felsenstein. Evolution 57, 1735-1746.

Gavrilets, S., 2000. Waiting time to parapatric speciation. Proc. R. Soc. London B 267, 2483-2492.

Gavrilets, S., 2004. Fitness Landscapes and the Origin of Species. Monographs in Population Biology, vol. 37. Princeton University Press, Princeton.

Gavrilets, S., Boake, C.R.B., 1998. On the evolution of premating isolation after a founder event. Am. Nat. 152, 706-716.
Gavrilets, S., Waxman, D., 2002. Sympatric speciation by sexual conflict. Proc. Natl. Acad. Sci. USA 99, 10533-10538.

Geritz, S.A.H., Kisdi, É., Meszéna, G., Metz, J.A.J., 1998. Evolutionarily singular strategies and the adaptive growth and branching of the evolutionary tree. Evol. Ecol. 12, 35-57.

Geritz, S.A.H., Gyllenberg, M., Jacobs, F.J.A, Parvinen, K., 2002. Invasion dynamics and attractor inheritance. J. Math. Biol. 44, 548-560.

Grant, P.G., Grant, R.B., 1997. Hybridization, sexual imprinting, and mate choice. Am. Nat. 149, 1-28.

Hauber, M.E., Pearson, H.E., Reh, A., Merges, A., 2002. Discrimination between host songs by brood parasitic brown-headed cowbirds Molothrus ater. Anim. Cogn. 5, 129-137.

Higashi, M., Takimoto, G., Yamamura, N., 1999. Sympatric speciation by sexual selection. Nature 402, 523-526.

Irwin, D.E., Price, T., 1999. Sexual imprinting, learning and speciation. Heredity 82, 347-354.

Karlin, S., McGregor, J., 1972. Application of method of small parameters to multi-niche population genetic models. Theor. Popul. Biol. 3, 186-209.

Karlin, S., McGregor, J., 1974. Towards a theory of the evolution of modifier genes. Theor. Popul. Biol. 5, 59-103.

Kawecki, T.J., 1997. Sympatric speciation via habitat specialization driven by deleterious mutations. Evolution 51, 1751-1763.

Kirkpatrick, M., 2001. Reinforcement during ecological speciation. Proc. R. Soc. London B 268, 1259-1263.

Kondrashov, A.S., 1986. Multilocus model of sympatric speciation. III. Computer simulations. Theor. Pop. Biol. 29, 1-15.

Kondrashov, A.S., Kondrashov, F.A., 1999. Interactions among quantitative traits in the course of sympatric speciation. Nature $400,351-354$.

Kondrashov, A.S., Mina, M.V., 1986. Sympatric speciation: when is it possible? Biol. J. Linn. Soc. 27, 201-223.

Laland, K.N., 1994a. On the evolutionary consequences of sexual imprinting. Evolution 48, 477-489.

Laland, K.N., 1994b. Sexual selection with a culturally transmitted mating preference. Theor. Popul. Biol. 45, 1-15.

Liou, L.W., Price, T.D., 1994. Speciation by reinforcement of premating isolation. Evolution 48, 1451-1459.

Lorenz, K., 1937. The companion in the bird's world. Auk 54, 245-273.

Maynard Smith, J., 1966. Sympatric speciation. Am. Nat. 100, 637-650.

Mayr, E., 1942. Systematics and the Origin of Species: From the Viewpoint of a Zoologist. Columbia University Press, New York.

Mayr, E., 1963. Animal Species and Evolution. Harvard University Press, Cambridge, MA.

Metz, J.A.J., Geritz, S.A.H., Meszéna, G., Jacobs, F.J.A., van Heerwaarden, J.S., 1996. Adaptive dynamics, a geometrical study of the consequences of nearly faithful reproduction. In: Van strien, D.H., Verduyn, S.M. (Eds.), Stochastic and Spatial Structures of Dynamical Systems. Elsevier, North-Holland, Amsterdam, pp. 147-194.

Owens, I.P., Rowe, C., Thomas, A.L., 1999. Sexual selection, speciation and imprinting: separating the sheep from the goats. Trends Ecol. Evol. 14, 131-132.

Payne, R.B., Payne, L.L., Woods, J.L., 1998. Song learning in broodparasitic indigobirds Vidua chalybeata: song mimicry of the host species. Anim. Behav. 55, 1537-1553.

Payne, R.B., Payne, L.L., Woods, J.L., Sorenson, M.D., 2000. Imprinting and the origin of parasite-host species associations in brood-parasitic indigobirds Vidua chalybeata. Anim. Behav. 59, 69-81.

Pimm, S.L., 1979. Sympatric speciation: a simulation model. Biol. J. Linn. Soc. 11, 131-139. 
Rice, W.R., 1984. Disruptive selection on habitat preference and the evolution of reproductive isolation: a simulation study. Evolution $38,1251-1260$.

Sadedin, S., Littlejohn, M.J., 2003. A spatially explicit individualbased model of reinforcement in hybrid zones. Evolution 57, 962-970.

Schilthuizen, M., 2001. Frogs, Flies, and Dandelions: Speciation-The Evolution of New Species. Oxford University Press, New York.

Servedio, M.R., 2000. Reinforcement and the genetics of nonrandom mating. Evolution 54, 21-29.

Servedio, M.R., Kirkpatrick, M., 1997. The effects of gene flow on reinforcement. Evolution 51, 1764-1772.

Servedio, M.R., Sætre, G.-P., 2003. Speciation as a positive feedback loop between postzygotic and prezygotic barriers to gene flow. Proc. R. Soc. London B 270, 1473-1479.

Slabbekoorn, H., Smith, T.B., 2002. Bird song, ecology and speciation. Philos. Trans. R. Soc. London B 357, 493-503. ten Cate, C., Bateson, P., 1988. Sexual selection: the evolution of conspicuous characteristics in birds by means of imprinting. Evolution 42, 1355-1358.

ten Cate, C., Vos, D.R., 1999. Sexual imprinting and evolutionary processes in birds: a reassessment. Adv. Study Behav. 28, $1-31$.

Thorpe, W.H., 1945. The evolutionary significance of habitat selection. Anim. Ecol. 14, 67-70.

Trickett, A.J., Butlin, R.K., 1994. Recombination suppressors and the evolution of new species. Heredity 73, 339-345.

Turelli, M., Barton, N.H., Coyne, J.A., 2001. Theory and speciation. Trends Ecol. Evol. 16, 330-343.

van Doorn, G.S., Weissing, F.J., 2001. Ecological versus sexual selection models of sympatric speciation: a synthesis. Selection 2, $17-40$.

West-Eberhard, M.J., 2003. Developmental Plasticity and Evolution. Oxford University Press, New York. 